\title{
Drug Repurposing of Lactoferrin Combination in a Nanodrug Delivery System to Combat Severe Acute Respiratory Syndrome Coronavirus-2 Infection
}

\author{
Assem Barakat ${ }^{1,2, *}(\mathbb{D})$, Abdullah Mohammed Al-Majid ${ }^{1,(\mathbb{D})}$, Gehad Lotfy ${ }^{3},\left(\mathbb{D}\right.$, Mohamed Ali ${ }^{1,(\mathbb{D}}$, \\ Ahmed Mostafa, ${ }^{4,(D)}$, Yaseen A.M.M. Elshaier ${ }^{5,(0)}$ \\ ${ }^{1}$ Department of Chemistry, College of Science, King Saud University, Riyadh, Saudi Arabia \\ ${ }^{2}$ Department of Chemistry, Faculty of Science, Alexandria University, Ibrahimia, Alexandria, Egypt \\ ${ }^{3}$ Department of Pharmacy, Aldara Hospital and Medical Center, Al Maazer, Riyadh, Saudi Arabia \\ ${ }^{4}$ Center of Scientific Excellence for Influenza Viruses, National Research Centre, Giza, Egypt \\ ${ }^{5}$ Department of Organic and Medicinal Chemistry, Faculty of Pharmacy, University of Sadat City, Menoufiya, Egypt
}

\section{ARTICLE INFO \\ Article History \\ Received 30 April 2021 \\ Accepted 02 August 2021 \\ Keywords \\ Drug repurposing \\ nanodrug delivery system \\ lactoferrin \\ nitazoxanide \\ spirooxindole \\ molecular modeling \\ SARS-CoV-2 \\ COVID-19}

\begin{abstract}
A drug repurposing approach for Food and Drug Administration (FDA)-approved drugs and preclinical entity by using cheminformatics and chemical transformational method, with the objective of discovering safer novel potent inhibitors that are selective for COVID-19, is reported. We examined the action of lactoferrin against Severe Acute Respiratory Syndrome Coronavirus-2 (SARS-CoV-2). Lactoferrin was tested not only as therapy but also as a nanocarrier. Among the different repurposed drugs approved to help control the COVID-19 pandemic, we focused on nitazoxanide in a nanodrug delivery form. Lactoferrin activity improved after it was used in combination with nitazoxanide [half-maximal inhibitory concentration $\left(\mathrm{IC}_{50}\right)=2.72,1.34$ with Selectivity Index $(\mathrm{SI})=25$ and 32, respectively]. These results will help us enhance the activity of lactoferrin as a nanocarrier for a variety of selected drugs. In addition, the antiviral activity of the spirooxindole scaffold showed interesting results against SARS-CoV-2 as well as Middle East respiratory syndrome (MERS)-CoV with an $\mathrm{IC}_{50}$ of 0.03 and $0.001 \mathrm{mM}$, respectively. The molecular modeling study revealed that nitazoxanide has a high similarity to arbidol. Docking outputs emphasize the importance of anilide functionality in drug activity because of its ability to form essential $\mathrm{HB}$ main protease and spike protein active sites. By understanding the binding mode, these data will help us design new bioactive drug candidates against SARS-CoV-2.
\end{abstract}

(C) 2021 Dr. Sulaiman Al Habib Medical Group. Publishing services by Atlantis Press International B.V. This is an open access article distributed under the CC BY-NC 4.0 license (http://creativecommons.org/licenses/by-nc/4.0/).

\section{INTRODUCTION}

A human coronavirus emerged in Wuhan, China (COVID-19) in late 2019 [1-3]. The World Health Organization (WHO) reported that, as of February 2, 2021, there have been 102,942,987 confirmed cases of COVID-19, including 2,232,233 deaths [4]. The symptoms caused by this viral infection including cough, fever, dyspnea, and lesion in the lungs [5]. In the advanced stage, the symptoms of this virus show pneumonia, which progresses to severe pneumonia and Acute Respiratory Distress Syndrome, which in turn results in the need for life support to sustain the patients $[6,7]$. The urgent need to discover new and repurposed drugs to treat this worldwide pandemic is highly challenging [8]. To reduce transmission and stop virus infection caused by COVID-19, the WHO suggested several crucial strategies; for example, isolating patients at early stages, minimizing social human-human contact, and wearing of masks. Recently several vaccines have been developed and used

"Corresponding author. Email: ambarakat@ksu.edu.sa

Peer review under responsibility of the Dr. Sulaiman Al Habib Medical Group

Data availability statement: The authors confirm that the data supporting the findings of this study are available within the article and/or its supplementary materials. under emergency purposes including Pfizer-BioNTech COVID-19 vaccine, Moderna COVID-19 vaccine, Oxford-AstraZeneca vaccine, and other vaccines developed by China and Russia; however, these vaccines are provided under crucial limitations [9]. To date, all developed vaccines or drugs used for COVID-19 treatment are suffering from many drawbacks.

The drug repurposing approach, one of the most efficient, facile, and promising protocol methods, was used to discover safer novel potent inhibitors that are selective for COVID-19. The reapplication of existing Food and Drug Administration (FDA)-approved therapeutic drugs to treat a new disease offers the advantages of rapid clinical impact at a lower cost and reduction of the number of steps involved in developing a new drug. Several factors favor the drug repurposing strategy, both at the preclinical and clinical stages. One of the typical scenarios in target-directed preclinical drug discovery is to initially focus on the optimization of binding affinity for the primary target, often with the simultaneous reduction of affinity for "secondary targets" (i.e., selectivity). Such efforts quite often leave aside the task of target profiling of said drug candidates for other, unrelated target classes, as well as drug pharmacokinetics and safety profiling [10]. 
Lactoferrin is a globular glycoprotein that originates in mammalian milk (Figure 1). It has a wide spectrum of medical and pharmacological activities, especially immunological activities. Many countries all over the world including those in the Middle East incorporated it in their protocols for treatment of COVID-19 patients. It has been reported that viruses initially attach to Heparan Sulfate Proteoglycans present in the surface of the host cell, and it has been shown that lactoferrin can inhibit this connection $[11,12]$.

To date, extensive efforts have been directed to repurpose FDAapproved drugs to combat Severe Acute Respiratory Syndrome Coronavirus-2 (SARS-CoV-2) using drug repurposing approaches. One of these drugs is nitazoxanide, an orally active broad-spectrum antiparasitic agent (Figure 1). Recent studies have shown its activity against SARS-CoV-2, but with low selective index and higher toxicity $[13,14]$.

Increasing selectivity while reducing toxicity is a challenging task. Nevertheless, when Barakat et al. $[15,16]$ engaged in a drug design and development research program, results indicated that the spirooxindole scaffold showed efficient anticancer properties with high selectivity and low toxicity, which are now under preclinical investigation.

Based on the finding mentioned above, we have assessed the antiviral activity of the following preclinical compounds and FDA-approved drugs including spirooxindole scaffold, lactoferrin, nitazoxanide, and also nitazoxanide-loaded lactoferrin nanoparticles using computational chemistry, molecular docking studies, and the shape similarity protocols for drug discovery for COVID-19 treatment.

\section{MATERIALS AND METHODS}

Absolute alcohol, dimethyl formamide, and glutaraldehyde solution (Sigma-Aldrich, Germany) were used.

\subsection{Synthesis of Spirooxindole}

The synthesis of the spirooxindole scaffold was prepared according to Barakat et al. $[15,16]$, and confirmation of the chemical structure was assigned and agreed with previously reported data.

\subsection{Nanoformulation of the Selected Drugs Lactoferrin and Nitazoxanide}

\subsubsection{Preparation of lactoferrin nanoparticles}

Lactoferrin nanoparticles were prepared according to previously reported protocols with several modifications [17,18]. In brief, a lactoferrin solution $(0.2 \% \mathrm{w} / \mathrm{v})$ was prepared by dissolving the appropriate amount in distilled water at Room Temperature (RT) for $1 \mathrm{~h}$. Then the $\mathrm{pH}$ of the solution was adjusted to 8. Afterward, the nanoprecipitation technique was followed through the dropwise addition of absolute alcohol into the lactoferrin solution. Finally, a few drops of glutaraldehyde solution and Tween 80 were added to ensure the crosslinking of the formed nanoparticles and to minimize their size, respectively [19].

\subsubsection{Preparation of nitazoxanide-loaded lactoferrin nanoparticles}

Lactoferrin and nitazoxanide were dissolved separately. For instance, lactoferrin was dissolved in distilled water as discussed in Section 2.2.1, whereas nitazoxanide was dissolved in dimethyl formamide. Next, alcohol and nitazoxanide solution were added dropwise at the same time into the lactoferrin solution, while stirring at RT. Finally, crosslinking of the nanoparticles as well as controlling their size were accomplished through adding glutaraldehyde and Tween 80 , respectively $[19,20]$.

\subsubsection{Particle size, polydispersity index, and zeta potential of prepared nanoparticles}

Particle size, Polydispersity Index (PDI), and zeta potential of each of the prepared nanoparticles were estimated through the Dynamic Light Scattering technique using Malvern Zetasizer (Malvern Instruments, Worcestershire, UK). The particle size analysis results contain both the $Z$-average mean and the PDI. PDI is estimated to investigate the uniformity of the particle size in the nanosuspension. A low PDI value indicates the narrow distribution of the particle size $[18,21]$. Meanwhile, zeta potential<smiles>O=C(/C=C/c1ccc(Cl)cc1Cl)C1[C@H](c2ccc(Cl)cc2Cl)C2CSCN2C12C(=O)Nc1ccccc12</smiles>

A potent and active MDM2-p53 inhibitor Barakat et al., US9822128B1

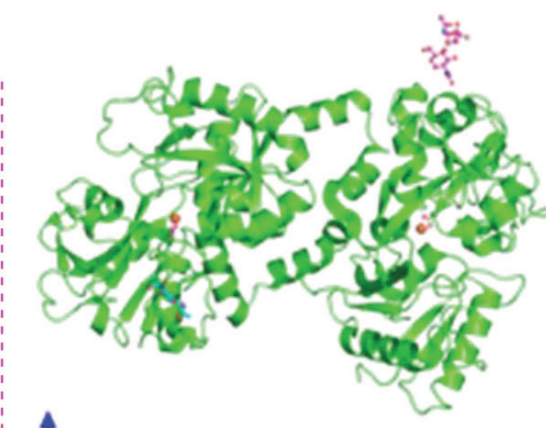

Lactoferrin<smiles>CC(=O)Oc1ccccc1C(=O)Nc1ncc([N+](=O)[O-])s1</smiles>

Nitazoxanide

Figure 1 Chemical structures of spirooxindole, lactoferrin, and nitazoxanide. 
is estimated to detect the surface charge of the prepared nanoparticles because the abundance of charges in nanoparticles prevent their aggregation, thereby increasing their stability within the nanosuspension $[17,19]$.

\subsection{Virology Study}

\subsubsection{Cytotoxicity $\left(\mathrm{CC}_{50}\right)$ determination}

To assess the half-maximal cytotoxic concentration $\left(\mathrm{CC}_{50}\right)$, stock solutions of the test compounds were prepared in $10 \%$ dimethyl sulfoxide in $\mathrm{ddH}_{2} \mathrm{O}$ and diluted further to the working solutions with Dulbecco's modified Eagle's medium [14,22-24]. The cytotoxic activity of the extracts was tested in Vero-E6 cells by using crystal violet assay as previously described [22] with minor modifications. Briefly, the cells were seeded in 96-well plates $(100 \mu \mathrm{L} /$ well at a density of $3 \times 10^{5}$ cells $/ \mathrm{mL}$ ) and incubated for $24 \mathrm{~h}$ at $37^{\circ} \mathrm{C}$ in $5 \% \mathrm{CO}_{2}$. After $24 \mathrm{~h}$, cells were treated with various concentrations of the tested compounds in triplicates. Seventy-two hours later, the supernatant was discarded, and cell monolayers were fixed with 10\% formaldehyde for $1 \mathrm{~h}$ at RT. The fixed monolayers are then dried and stained with $50 \mu \mathrm{L}$ of $0.1 \%$ crystal violet for $20 \mathrm{~min}$ on a bench rocker at RT. The monolayers are then washed and dried, and the crystal violet dye in each well is then dissolved with $200 \mu \mathrm{L}$ methanol for 20 min on a bench rocker at RT. Absorbance of crystal violet solutions is measured at $\lambda_{\max } 570 \mathrm{~nm}$ as a reference wavelength using a multiwell plate reader. The percentage of cytotoxicity compared to the untreated cells was determined with the following equation.

The plot of \% cytotoxicity versus sample concentration was used to calculate the concentration which exhibited $50 \%$ cytotoxicity $\left(\mathrm{CC}_{50}\right)[24]$.

$$
\% \text { Cytotoxicity }=\frac{\begin{array}{l}
\text { Absorbance of cells without treatment }- \\
\text { Absorbance of cells with treatment }) \times 100
\end{array}}{\text { Absorbance of cells without treatment }}
$$

\subsubsection{Inhibitory concentration determination}

The half-maximal inhibitory concentration $\left(\mathrm{IC}_{50}\right)$ values for the tested compounds were determined as previously described [14], with minor modifications. Briefly, in 96-well tissue culture plates,
$2.4 \times 10^{4}$ Vero-E6 cells were distributed in each well and incubated overnight at a humidified $37^{\circ} \mathrm{C}$ incubator under $5 \% \mathrm{CO}_{2}$ condition. The cell monolayers were then washed once with $1 \times$ Phosphatebuffered Sulfate (PBS). An aliquot of the SARS-CoV-2 "NRC-03nhCoV" virus [23] containing $100 \mathrm{TCID}_{50}$ was incubated with serial diluted compounds and kept at $37^{\circ} \mathrm{C}$ for $1 \mathrm{~h}$. The Vero-E6 cells were treated with the virus-compound mix and coincubated at $37^{\circ} \mathrm{C}$ in a total volume of $200 \mu \mathrm{L} /$ well. Untreated cells infected with virus represent virus control; however, cells that are not treated and not infected served as cell control. After incubation at $37^{\circ} \mathrm{C}$ in $5 \% \mathrm{CO}_{2}$ incubator for $72 \mathrm{~h}$, the cells were fixed with $100 \mu \mathrm{L}$ of $10 \%$ paraformaldehyde for $20 \mathrm{~min}$ and stained with $0.5 \%$ crystal violet in distilled water for $15 \mathrm{~min}$ at RT. The crystal violet dye was then dissolved using $100 \mu \mathrm{L}$ absolute methanol per well, and the optical density of the color is measured at $570 \mathrm{~nm}$ using Anthos Zenyth 200rt plate reader (Anthos Labtec Instruments, Heerhugowaard, The Netherlands). The $\mathrm{IC}_{50}$ of the compound is that required to reduce the virus-induced Cytopathic Effect (CPE) by 50\%, relative to the virus control.

\subsection{Molecular Modeling}

The molecular docking and shape similarity known as Rapid Overlay Chemical Structure (ROCS) studies were operated using the OpenEye Modeling software [Fast Rigid Exhaustive Docking Receptor, version 2.2.5; OpenEye Scientific Software, SantaFe, NM, USA; http://www.eyesopen.com]. A virtual library of the target drugs was used, and their energies were minimized using the MMFF94 force field, followed by the generation of multiconformers using the OMEGA application. Data were visualized by Vida commands.

\section{RESULTS AND DISCUSSION}

\subsection{Synthesis of Spirooxindole Scaffold}

Our attention was directed to examine the activity of a patented compound with anticancer activity that originated from chemical architecture, namely, spirooxonindoles $[15,16]$. The drug candidate was synthesized in a large-scale quantity for antiviral activity according to the method described by Barakat et al. $[15,16]$, as shown in Figure 2. The multicomponent reaction of dienone with thioproline and isatin in $\mathrm{MeOH}$ under heating for $1.5-2 \mathrm{~h}$ at $60-65^{\circ} \mathrm{C}$ was utilized to afford the target spirooxindole scaffold.<smiles>C=Cc1ccc(Cl)cc1Cl</smiles>

Thioproline

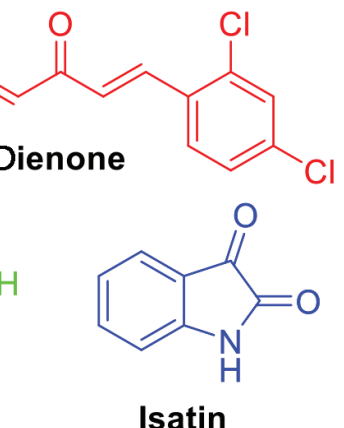<smiles>[H][R9]([H])(OC)O[Na]</smiles>

Spirooxindole scaffold

Figure 2 Synthesis of the spirooxindole scaffold. 


\subsection{Preparation and Characterization of Lactoferrin-based Nanoparticles}

Both lactoferrin and nitazoxanide-loaded lactoferrin nanoparticles were successfully prepared. This was observed through the formation of slightly turbid and stable nanosuspensions of both nanoparticles. Furthermore, the results of Zetasizer measurements and Scanning Electron Microscopy (SEM) confirmed the formation of both particles at the nanoscale range. Furthermore, Fourier Transform Infrared (FTIR) analysis confirmed the formation of nitazoxanide-loaded lactoferrin nanoparticles.

\subsection{Particle size analyzer}

Lactoferrin nanoparticles showed an average size of $345 \pm 12.5 \mathrm{~nm}$ with a PDI of 0.225 . This is considered to be small enough to be engulfed by cells crossing their membranes. In addition, the zeta potential for the prepared nanoparticles was found to be $-15.1 \pm$ $1.9 \mathrm{mV}$. This charge is considered an advantage to guarantee the repulsion between nanoparticles, thus preventing their aggregation $[17,18]$. Moreover, nitazoxanide-loaded lactoferrin nanoparticles possess an average size of $353 \pm 17 \mathrm{~nm}$ and a PDI of 0.195 . This is also a good particle size to be able to penetrate the cell membrane [25]. Furthermore, the zeta potential for the prepared nanoparticles was recorded to be $-13.9 \pm 2.4 \mathrm{mV}$. This is still considered enough surface charge to prevent aggregation of nanoparticles. Therefore, they are considered to form stable nanosuspension $[17,18]$. Finally, the nanoparticle showed an entrapment efficiency of almost $70 \%$ of the loaded drug.

In conclusion, it is observed that the loading of nitazoxanide within the lactoferrin matrix did not significantly affect their particle size or their surface charge. Therefore, it is concluded that nitazoxanide can be loaded successfully within lactoferrin nanoparticles without negatively affecting their efficacy to penetrate cell membranes or to form stable nanosuspension.

\subsubsection{SEM analysis}

As shown in Figure 3A and 3B, SEM images of both lactoferrin and nitazoxanide-loaded lactoferrin nanoparticles indicate that they have spherical shapes with an average particle size of $320-340 \mathrm{~nm}$ for lactoferrin nanoparticles and 335-370 nm for nitazoxanideloaded lactoferrin nanoparticles, confirming the results of the particle size analyzer.

\subsubsection{FTIR analysis}

Fourier transform infrared analysis was used for in-depth examination of the chemical composition of the pristine materials as well as the as-fabricated composite. The FTIR spectrum (Figure 4A) clearly reveals the main distinctive bands to lactoferrin as the absorption bands at 1662,1528 , and $1093 / \mathrm{cm}$ are ascribed to amide I, amide II, and C-O-C, respectively [26,27]. It is well known that amide I is more easily distinguishable from the secondary structure ( $\alpha$-helix, $\beta$-sheet, etc.), which is most likely attributable to $\mathrm{C}=\mathrm{O}$ stretching vibrations of the peptide bonds [28]. Moreover, the

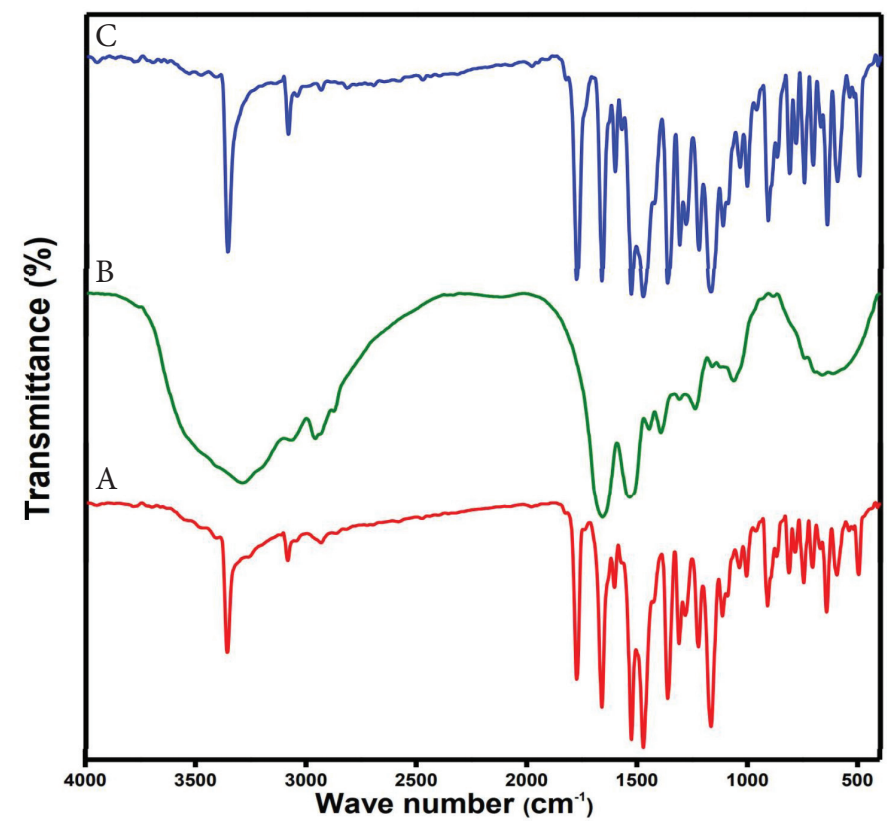

Figure 4 FTIR of (A) lactoferrin, (B) nitazoxanide, and (C) nitazoxanideloaded lactoferrin nanoparticles.
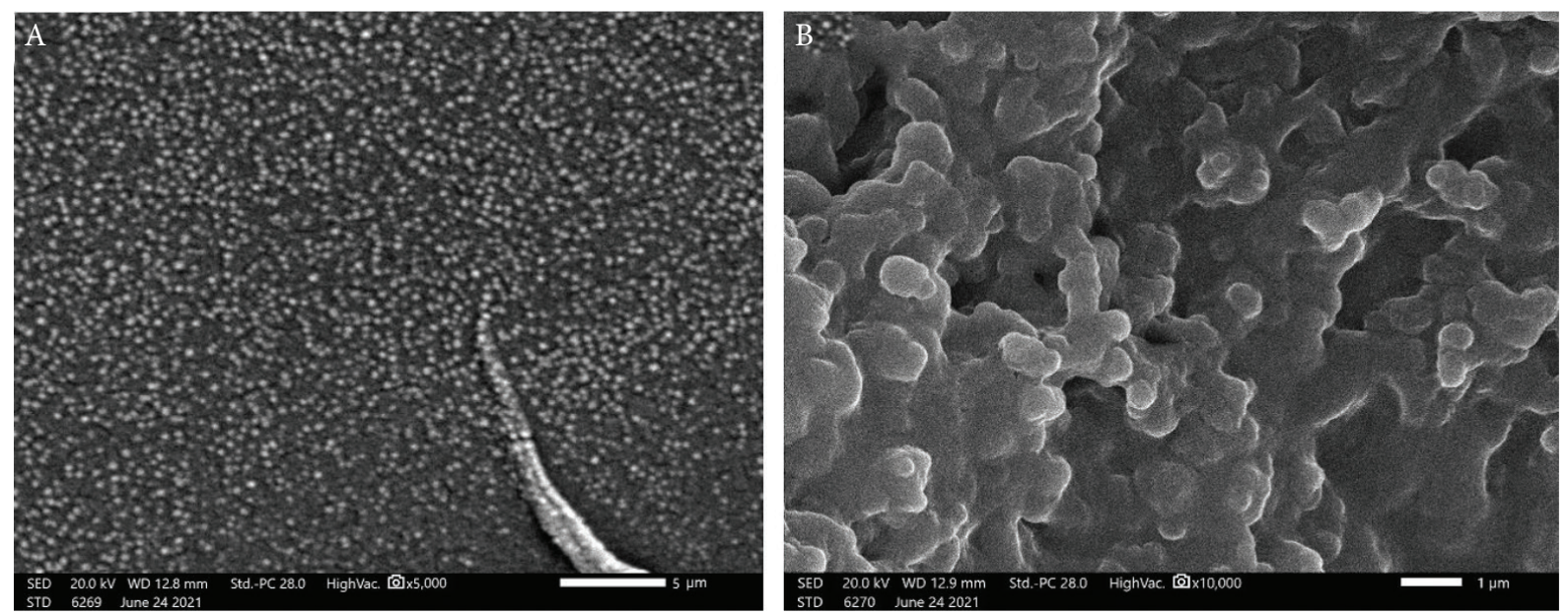

Figure 3 SEM images of (A) lactoferrin and (B) nitazoxanide-loaded lactoferrin nanoparticles. 
FTIR spectrum of nitazoxanide (Figure 4B) shows the characteristic peaks of the carbonyl group of amide and ester linkages at 1536 and $1659 / \mathrm{cm}$, respectively [29]. Furthermore, the two peaks at 1448 and $3069 / \mathrm{cm}$ are attributed to nitro group and $=\mathrm{CH}$, respectively [30]. The FTIR spectrum (Figure 4C) depicts the characteristic bands of lactoferrin and nitazoxanide, as well as the increase in peak intensity of carbonyl groups at $1536 / \mathrm{cm}$, indicating the successful conjugation between lactoferrin and nitazoxanide [31].

\subsection{Antiviral Activity}

\subsubsection{Antiviral activity of the patented spirooxindole as a drug candidate}

The antiviral activity of the spirooxindole scaffold showed interesting results against SARS-CoV-2 as well as Middle East Respiratory Syndrome (MERS)-CoV with $\mathrm{IC}_{50}=0.03$ and $0.001 \mathrm{mM}$, respectively (Figure 5).

\subsubsection{Antiviral activity of lactoferrin, nitazoxanide, and nitazoxanide- loaded lactoferrin nanoparticles}

The antiviral activities of lactoferrin and the lactoferrin/nitazoxanide composite were experimentally assessed using nontoxic concentrations (Figure 6A). Interestingly, the nitazoxanide improved the $\mathrm{IC}_{50}\left(\mathrm{IC}_{50}=2.273\right.$ for lactoferrin, $\mathrm{IC}_{50}=1.438$ for the lactoferrin/ nitazoxanide composite) and the Selectivity Index (SI) of lactoferrin (approximately SI = 25 for lactoferrin and SI $=32$ for lactoferrin/nitazoxanide). This result indicates the synergistic effect of the composite components. This finding will open the gates for the use of lactoferrin drug in the future as a drug delivery system to target the lung and stop cytokine storm in COVID-19 patients.

\subsection{Molecular Modeling Study}

\subsubsection{Shape similarity and molecular alignment}

Rapid overlay chemical structure is a virtual screening technique used to detect resemblance between organic molecules based on their Three-Dimensional (3D) shapes [32].
High similarity in shape reflects high similarity in biological activity whereas high similarity in biological activity does not reflect the relationship between biological activity and the 3D shape structures. ROCS can be used in different applications such as virtual screening, lead hopping, molecular alignment, pose generation, and drug repurposing $[33,34]$. The tactic of this study is to recognize the similarity of nitazoxanide to different drugs with known activity against SARS-COV-2 [35,36].

Rapid overlay chemical structure analysis includes two files, which must be in the most stable conformer generated by Omega algorism: (1) nitazoxanide as database and (2) query file, which refers to the selected drugs or standard ligands. With this study, we can figure out the most possible mechanism in targeting SARS-CoV-2 for nitazoxanide. The study processed matches between nitazoxanide and query drugs in an isolated run. This match is based on volume overlap of optimally aligned molecules, which are virtually independent of the atom types and bonding patterns of the query. Two main outputs were produced from the ROCS study. The first one is shape similarity, which includes shape counter, shape atoms, and color atom labels. Figure 7A displays umifenovir with three rings, two acceptors, one donor, and hydrophobe. Nitazoxanide overlay with umifenovir is shown in Figure $7 \mathrm{~B}$, showing high similarity. Then we examined the color and shape of nitazoxanide. Figure $7 \mathrm{C}$ shows two rings, five acceptors, and one donor.

The second output is a set of scores expressed in Tanimoto scores. The most imperative score is the Tanimoto Combo (TC), which measures shape fit and color fit. This has a value between 0 and 2, and the score is used for ranking the hit list (Table 1).

In the ROCS analysis for nitazoxanide against different queries, Table 1 shows that nitazoxanide has TC scores to umifenovir, fingolimod, remdesiver, ketoamide, N3, and lopinavir. In this regard and in reference to the molecular mechanisms for these known queries (Figure 8), nitazoxanide is expected to have activity against spike glycoprotein to prevent virus entry or may have preventive activity toward inflammatory storm in COVID-19 patients. In this respect, we envisioned to combine this drug with lactoferrin.

\subsubsection{Docking study}

We used the docking approach to examine the binding mode of nitazoxanide with the different molecular pathways of SARS-CoV-2 during its replication including main protease $\left(\mathrm{M}^{\mathrm{pro}}\right)$, RNA polymerase, and spike glycol proteins. Nitazoxanide
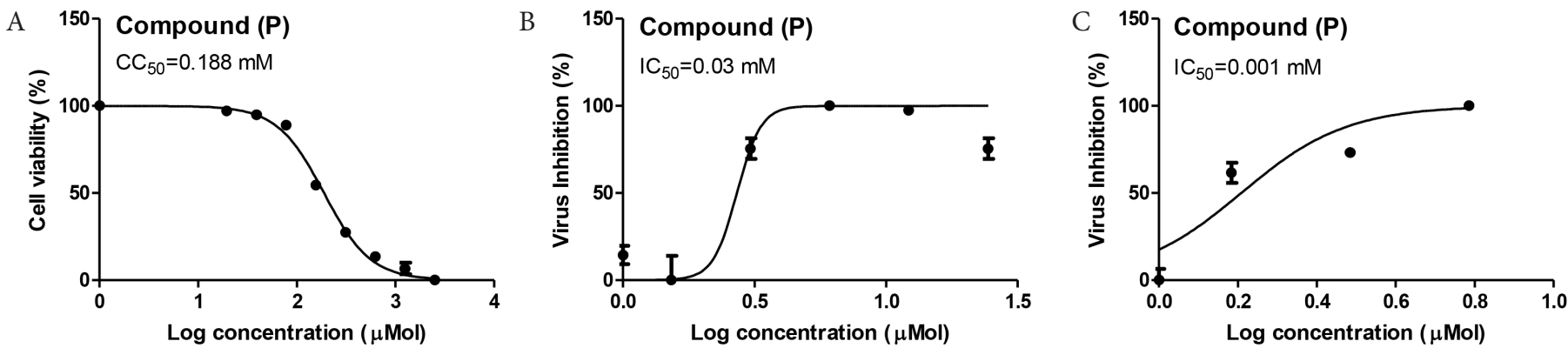

Figure 5 Antiviral activity of the spirooxindole. (A) Half-maximal cytotoxic concentration $\left(\mathrm{CC}_{50}\right)$ of the tested compounds in Vero-E6 cells. (B) Halfmaximal inhibitory concentration $\left(\mathrm{IC}_{50}\right.$ ) of the tested compound against NRC-03-nhCoV virus in Vero-E6 cells (testing against SARS-CoV-2). (C) IC ${ }_{50}$ against MERS-CoV. $\mathrm{IC}_{50}$, half-maximal inhibitory concentration. 

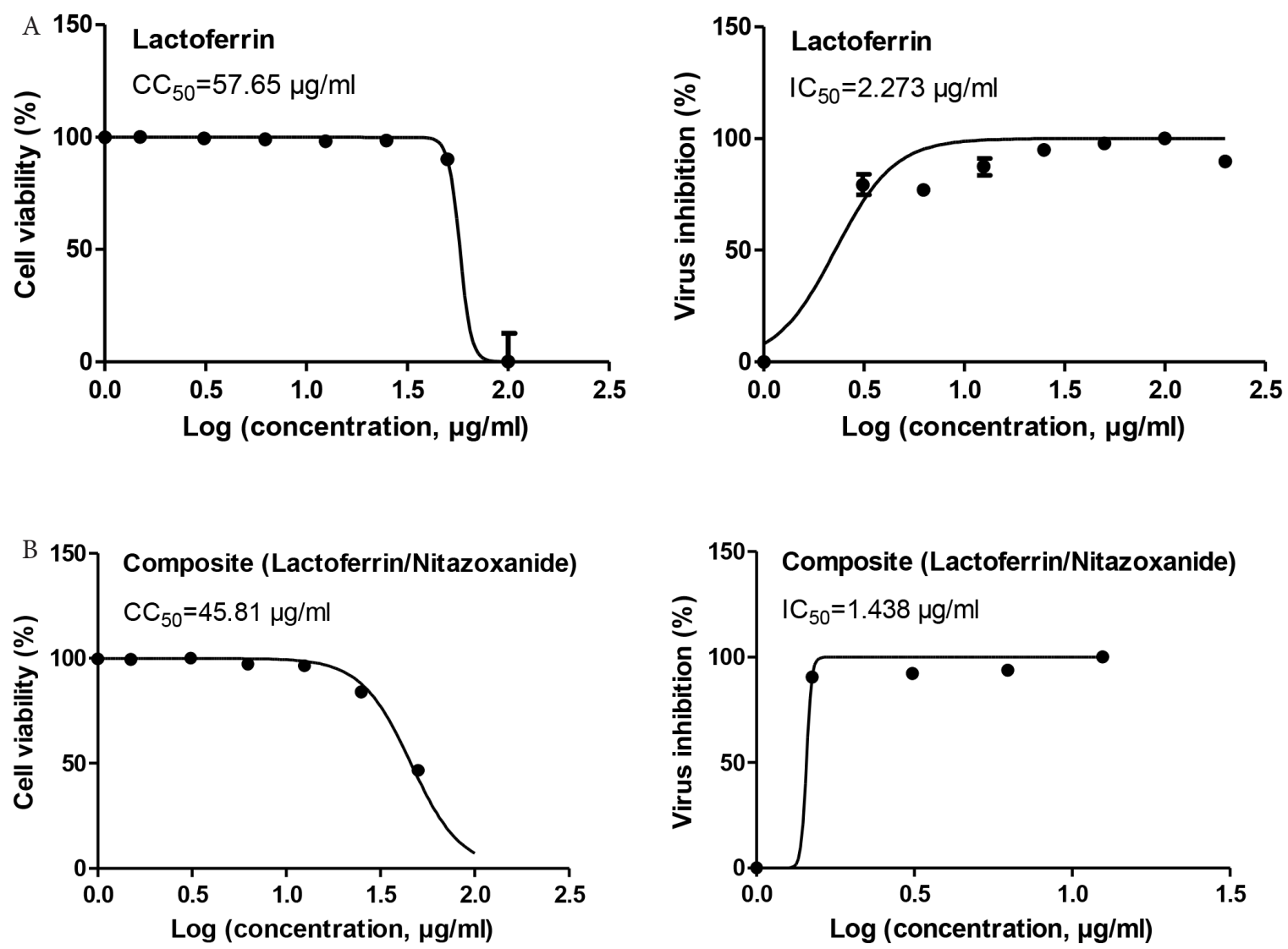

Figure 6 Antiviral testing against (SARS-CoV-2). (A) Half maximal cytotoxic concentration $\left(\mathrm{CC}_{50}\right.$ ) of the tested compounds in Vero-E6 cells. (B) Halfmaximal inhibitory concentration $\left(\mathrm{IC}_{50}\right)$ of the tested compounds against NRC-03-nhCoV virus in Vero-E6 cells. Data are presented as the average of the means. The $\mathrm{CC}_{50}$ and $\mathrm{IC}_{50}$ curves represent the nonlinear fit of "Normalize" of "Transform" of the obtained data. Their values were calculated using GraphPad Prism (version 5.01) as "best-fit value." $\mathrm{CC}_{50}$, half-maximal cytotoxic concentration.
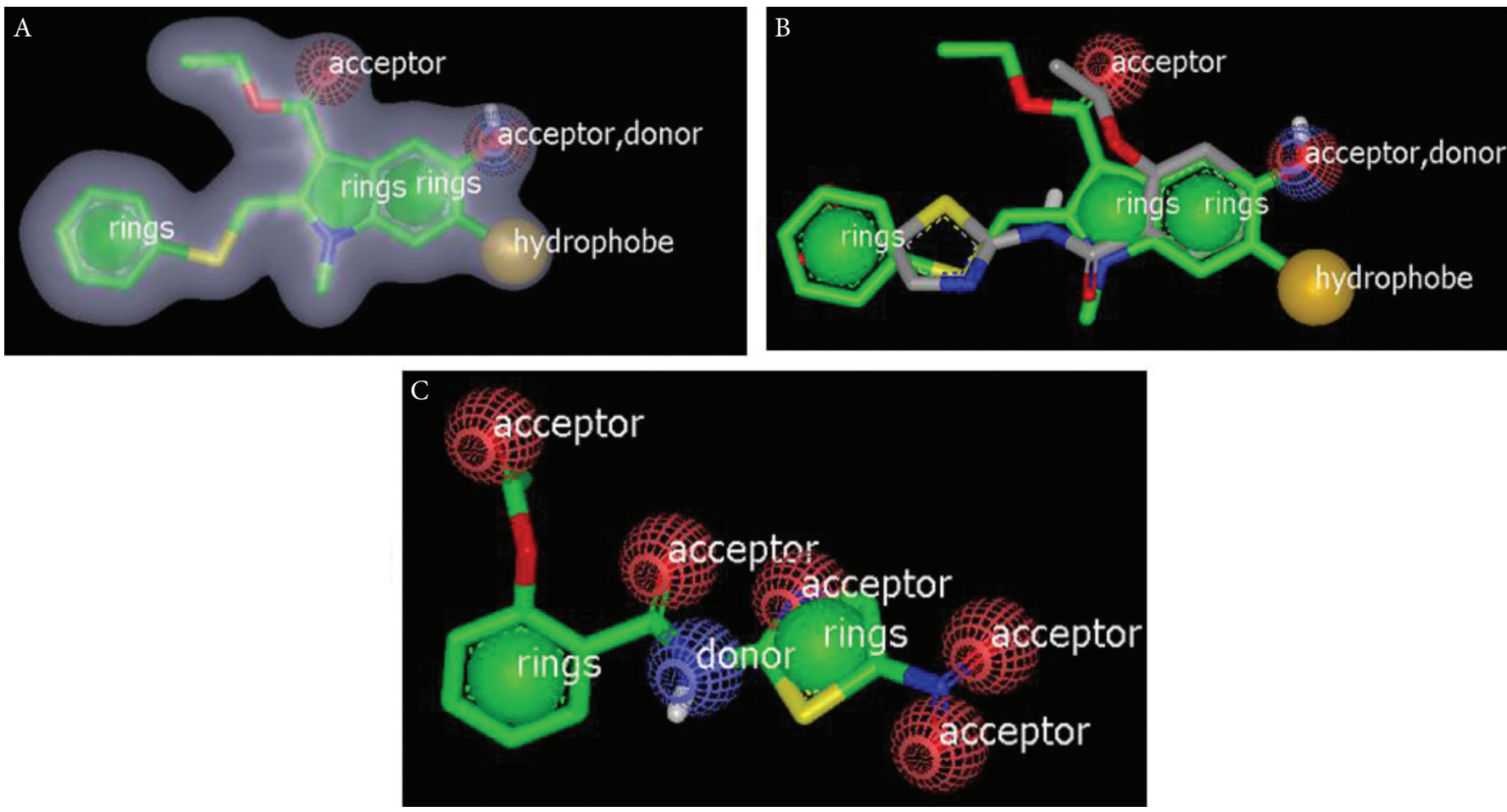

Figure 7 (A) Umifenovir color volume and shape. (B) Nitazoxanide (grey color) overlay with umifenovir (green). (C) Nitazoxanide color volume and shape. 
Table 1 Tanimoto combo scores of different drugs recommended in COVID-19 disease

\begin{tabular}{|c|c|c|}
\hline Drugs & TC score & Mode of action against SARS-CoV-2 \\
\hline$\alpha$-Ketoamide & 0.52 & $\begin{array}{l}\text { Cocrystalized ligand of SARS-CoV-2 } \\
\text { M }^{\text {pro }} \text { (PDB ID: 6Y2F) }\end{array}$ \\
\hline N3 & 0.48 & $\begin{array}{l}\text { Cocrystalized ligand of SARS-CoV-2 } \\
\text { M }^{\text {pro }} \text { (PDB ID: 6LU7) }\end{array}$ \\
\hline Remdesivir & 0.54 & $\begin{array}{l}\text { Inhibiting the RNA-dependent RNA } \\
\text { polymerase }\end{array}$ \\
\hline Lopinavir & 0.46 & Inhibiting the main protease \\
\hline Umifenovir (Arbidol) & 0.78 & Blocking virus-cell membrane fusion \\
\hline Fingolimod & 0.72 & Preventing the inflammatory storm \\
\hline
\end{tabular}

occupied the active site of $\mathrm{M}^{\text {pro }}$ (PDB ID: 6y2f) with formation of HB with Met:165A through the hydrogen of anilide moiety. The other pharmacophoric features of nitazoxanide interacted without formation of $\mathrm{HB}$ (Figure 9A). Figure 9B displays the binding mode and pose of nitazoxanide with spike protein (PDB ID: 6vsb). Again, the hydrogen of $\mathrm{NH}$ participates in $\mathrm{HB}$ with the receptor amino acid, ASN: 422B. Nitazoxanide interaction with RNA polymerase receptor (PDB ID: 6m71) exhibited a different binding mode. The nitro functionality participates in HB with THR:76 A (Figure 9C).

\section{CONCLUSION}

In this study, we used the bioinformatics approach along with the antiviral activity of spirooxindole scaffold, lactoferrin, nitazoxanide, and nitazoxanide loaded into lactoferrin nanoparticles. The results showed improvement in the activity and SI of nitazoxanide/lactoferrin nanoparticles $\left(\mathrm{IC}_{50}=1.438 \mu \mathrm{M} ; \mathrm{SI}=32\right)$ compared with lactoferrin $\left(\mathrm{IC}_{50}=2.273 \mu \mathrm{M}\right.$; SI = 25). With its high antiviral activity, spirooxindole could be considered as a lead compound for drug discovery and development to combat
A<smiles>Cc1cccc(C)c1OCC(=O)NC(Cc1ccccc1)[C@@H](O)CC(Cc1ccccc1)NC(=O)[C@@H](C(C)C)N1CCCNC1=O</smiles>

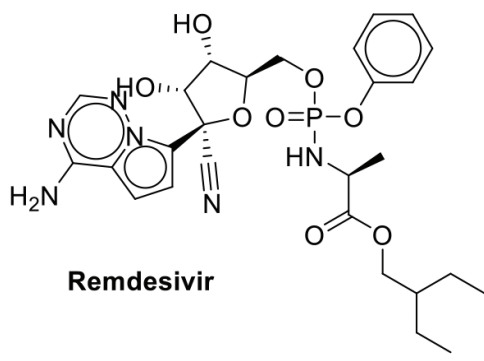<smiles>[R10]C(C)[C@H](NC(=O)N(C)Cc1csc(C(C)C)n1)C(=O)N[C@@H](Cc1ccccc1)C[C@@H](O)[C@H](Cc1ccccc1)NC(=O)OCc1cncs1</smiles><smiles>CCOC(=O)c1c(CSc2ccccc2)n(C)c2cc(Br)c(O)c(CN(C)C)c12</smiles>

Umifenovir

B<smiles>Cc1cc(C(=O)N[C@@H](C)C(=O)N[C@H](C(=O)N[C@@H](CC(C)C)C(=O)N[C@@H](/C=C/C(=O)OCc2ccccc2)C[C@@H]2CCNC2=O)C(C)C)no1</smiles>

Ligand 1

Figure 8 Chemical structures. (A) Reported drugs prescribed in COVID-19 cases. (B) Ligands N3 and $\alpha$-ketoamide for Severe Acute Respiratory Syndrome Coronavirus-2 (SARS-CoV-2) M ${ }^{\text {pro }}$ (PDB ID: 6LU7 and 6Y2F, respectively [37,38]) and ligand 1 for SARS-CoV-2 spike protein (PDB ID: 6vsb) [39]. 
A

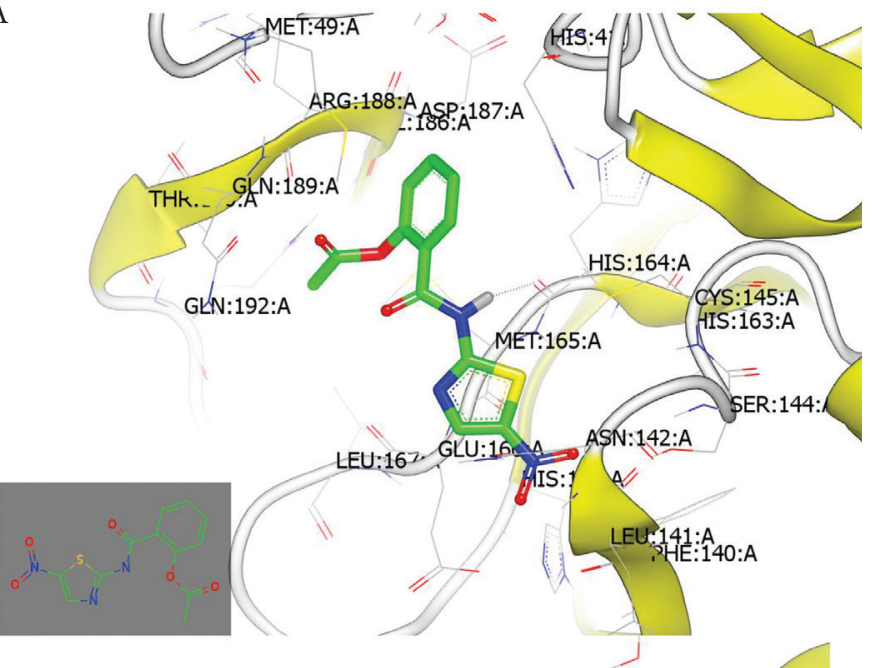

B

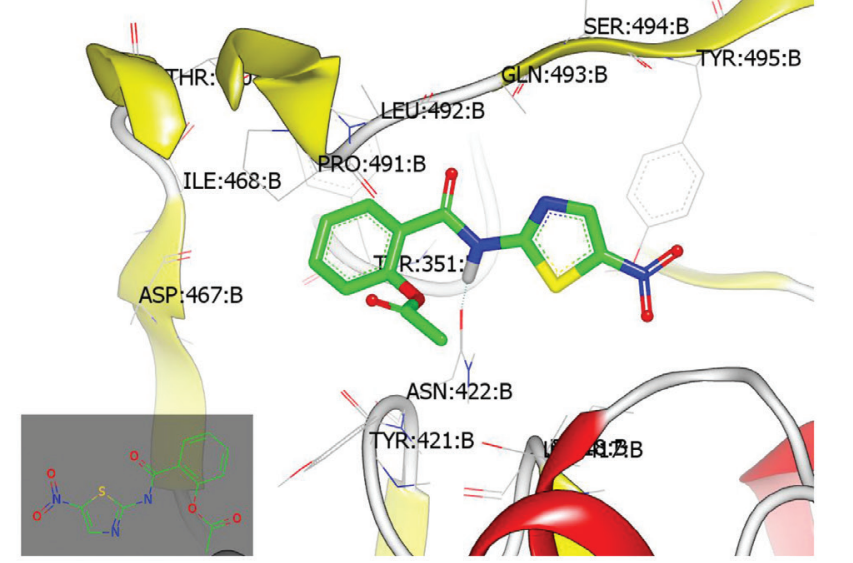

C

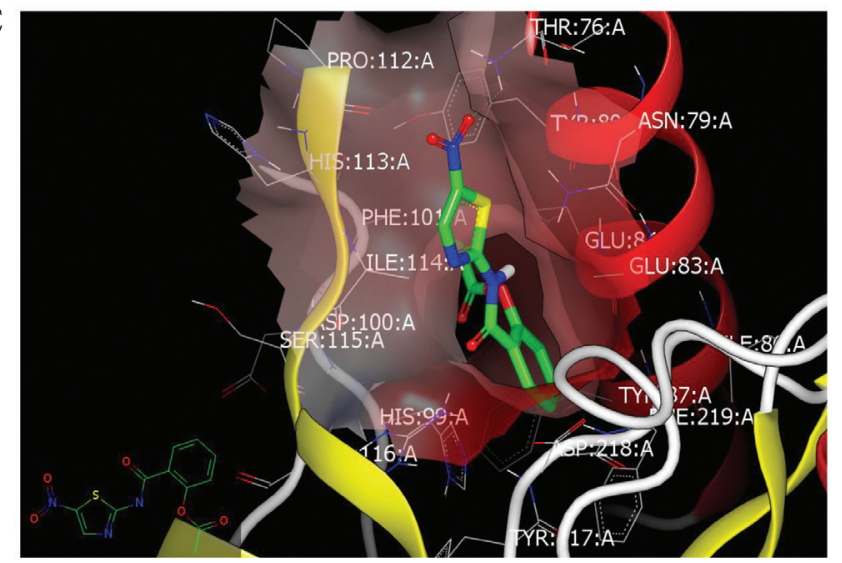

Figure 9 (A) Nitazoxanide with $\mathrm{M}^{\text {pro }}$ (PDB ID: 6y2f); (B) with spike protein (PDB ID: 6vsb); (C) with RNA polymerase (PDB ID: 6m71).

MERS-CoV. Finally, we suggest using lactoferrin as a nanodrug delivery system for nitazoxanide as part of preclinical investigation to fight against COVID-19.

\section{CONFLICTS OF INTEREST}

The authors declare no conflict of interest, and the funding body had no role in study design, data collection, or data interpretation in the study.

\section{AUTHORS' CONTRIBUTION}

$\mathrm{AB}$ contributed in study conceptualization and writing (review and editing) the manuscript. AMA-M, GE and MA contributed in data curation, and formal analysis writing (original draft). AM contributed in antiviral activity analysis. YAMME contributed in bioinformatics studies for discovery of the repurposed drug. $\mathrm{AB}$ contributed in funding acquisition and project administration. All authors helped in writing (original draft) the manuscript, editing, and approval of the final form of the manuscript.

\section{ACKNOWLEDGMENTS}

The authors thank the Dr Sulaiman Al Habib Medical Services Group Company for providing funding to this research project.

\section{REFERENCES}

[1] Coronaviridae Study Group of the International Committee on Taxonomy of Viruses. The species Severe acute respiratory syndrome-related coronavirus: classifying 2019-nCoV and naming it SARS-CoV-2. Nat Microbiol 2020;5;536-44.

[2] Kupferschmidt K, Cohen J. Will novel virus go pandemic or be contained?. Science 2020;367;610-11.

[3] Mirza MU, Froeyen M. Structural elucidation of SARS-CoV-2 vital proteins: computational methods reveal potential drug candidates against main protease, Nsp12 polymerase and Nsp13 helicase. J Pharm Anal 2020;10;320-8.

[4] WHO Coronavirus (COVID-19) Dashboard. Available from: https://covid19.who.int/ (accessed February 2, 2021).

[5] Huang C, Wang Y, Li X, Ren L, Zhao J, Hu Y, et al. Clinical features of patients infected with 2019 novel coronavirus in Wuhan, China. Lancet 2020;395;497-506.

[6] Wu F, Zhao S, Yu B, Chen YM, Wang W, Song ZG, et al. A new coronavirus associated with human respiratory disease in China. Nature 2020;579;265-9.

[7] Weiss SR, Navas-Martin S. Coronavirus pathogenesis and the emerging pathogen severe acute respiratory syndrome coronavirus. Microbiol Mol Biol Rev 2005;69;635-64.

[8] Chen B, Liu M, Huang C. Current diagnostic and therapeutic strategies for COVID-19. J Pharm Anal 2021;11;129-137.

[9] Li T, Zhang, T, Gu Y, Li S, Xia N. Current progress and challenges in the design and development of a successful COVID-19 vaccine. Fundam Res 2021;1;139-150.

[10] Batalha PN, Forezi LSM, Lima CGS, Pauli FP, Boechat FCS, de Souza MCBV, et al. Drug repurposing for the treatment of COVID-19: pharmacological aspects and synthetic approaches. Bioorg Chem 2021;106;104488.

[11] Kell DB, Heyden EL, Pretorius E. The biology of lactoferrin, an iron-binding protein that can help defend against viruses and bacteria. Front Immunol 2020;11;1221.

[12] Allam AE, Assaf HK, Hassan HA, Shimizu K, Elshaier YAMM. An in silico perception for newly isolated flavonoids from peach fruit as privileged avenue for a countermeasure outbreak of COVID-19. RSC Adv 2020;10;29983-98.

[13] Kelleni MT. Nitazoxanide/azithromycin combination for COVID-19: a suggested new protocol for early management. Pharmacol Res 2020;157;104874. 
[14] Mostafa A, Kandeil A, Elshaier YAMM, Kutkat O, Moatasim Y, Rashad AA, et al. FDA-approved drugs with potent in vitro antiviral activity against severe acute respiratory syndrome coronavirus 2. Pharmaceuticals 2020;13;443.

[15] Barakat A, Islam MS, Ghawas HM, Al-Majid AM, El-Senduny FF, Badria FA, et al. Design and synthesis of new substituted spirooxindoles as potential inhibitors of the MDM2-p53 interaction. Bioorg Chem 2019;86;598-608.

[16] Barakat A, Islam MS, Al-Majid AM, Ghawas HM, El-Senduny FF, Badria FA, et al. Substituted spirooxindoles. King Saud University, 2017. U.S. Patent 9,822,128. Available from: https:// patents.google.com/patent/US9822128B1/en.

[17] Martins JT, Santos SF, Bourbon AI, Pinheiro AC, GonzálezFernández A, Pastrana LM, et al. Lactoferrin-based nanoparticles as a vehicle for iron in food applications - development and release profile. Food Res Int 2016;90;16-24.

[18] Mofidian R, Barati A, Jahanshahi M, Shahavi MH. Optimization on thermal treatment synthesis of lactoferrin nanoparticles via Taguchi design method. SN Appl Sci 2019;1;1339.

[19] Hamdi M, Abdel-Bar HM, Elmowafy E, Al-Jamal KT, Awad GAS. An integrated vitamin E-coated polymer hybrid nanoplatform: a lucrative option for an enhanced in vitro macrophage retention for an anti-hepatitis B therapeutic prospect. PLoS One 2020;15;e0227231.

[20] Zhang H, Shi Y, Wang H. Solubility determination of nitazoxanide in twelve organic solvents from $T=273.15$ to $313.15 \mathrm{~K}$. J Chem Eng Data 2020;65;3645-51.

[21] Quiñones JP, Brüggemann O, Kjems J, Shahavi MH, Covas CP. Novel brassinosteroid-modified polyethylene glycol micelles for controlled release of agrochemicals. J Agric Food Chem 2018;66;1612-19.

[22] Feoktistova M, Geserick P, Leverkus M. Crystal violet assay for determining viability of cultured cells. Cold Spring Harb Protoc 2016;2016;343-6.

[23] Kandeil A, Mostafa A, El-Shesheny R, Shehata M, Roshdy WH, Ahmed SS, et al. Coding-complete genome sequences of two SARS-CoV-2 isolates from Egypt. Microbiol Resour Announc 2020;9;e00489-20.

[24] Mosmann T. Rapid colorimetric assay for cellular growth and survival: application to proliferation and cytotoxicity assays. J Immunol Methods 1983;65;55-63.

[25] Shang L, Nienhaus K, Nienhaus GU. Engineered nanoparticles interacting with cells: size matters. J Nanobiotechnol 2014; $12 ; 5$.

[26] Kumar P, Lakshmi YS, Bhaskar C, Golla K, Kondapi AK. Improved safety, bioavailability and pharmacokinetics of zidovudine through lactoferrin nanoparticles during oral administration in rats. PLoS One 2015;10;e0140399.

[27] Duca G, Anghel L, Erhan RV. Structural aspects of lactoferrin and serum transferrin observed by FTIR spectroscopy. Chem J Mold 2018;13;111-16.

[28] Ono K, Sakai H, Tokunaga S, Sharmin T, Aida TM, Mishima K. Encapsulation of lactoferrin for sustained release using particles from gas-saturated solutions. Processes 2021;9;73.

[29] Ali NW, Abbas SS, Zaazaa HES, Abdelrahman MM, Abdelkawy M. Validated stability indicating methods for determination of nitazoxanide in presence of its degradation products. J Pharm Anal 2012;2;105-16.

[30] Sachan AK, Gupta A. Formulation and evaluation of bilayer tablets of nitazoxanide. Der Pharm Lett 2017;9;1-9.

[31] Luo B, Liang H, Zhang S, Qin X, Liu X, Liu W, et al. Novel lactoferrin-conjugated amphiphilic poly(aminoethyl ethylene phosphate)/poly (L-lactide) copolymer nanobubbles for tumortargeting ultrasonic imaging. Int J Nanomedicine 2015;10;5805-17.

[32] Mahmoud DB, Shitu Z, Mostafa A. Drug repurposing of nitazoxanide: can it be an effective therapy for Covid-19?. J Genet Eng Biotechnol 2020;18;35.

[33] Wang M, Cao R, Zhang L, Yang X, Liu J, Xu M, et al. Remdesivir and chloroquine effectively inhibit the recently emerged novel coronavirus (2019-nCoV) in vitro. Cell Res 2020;30;269-71.

[34] Elbastawesy MAI, El-Shaier YAMM, Ramadan M, Brown AB, Aly AA, Abuo-Rahma GE-DA. Identification and molecular modeling of new quinolin-2-one thiosemicarbazide scaffold with antimicrobial urease inhibitory activity. Mol Divers $2021 ; 25 ; 13-27$.

[35] Rush TS, Grant JA, Mosyak L, Nicholls A. A shape-based 3-D scaffold hopping method and its application to a bacterial protein-protein interaction. J Med Chem 2005;48;1489-95.

[36] Lee HS, Choi J, Kufareva I, Abagyan R, Filikov A, Yang Y, et al. Optimization of high throughput virtual screening by combining shape-matching and docking methods. J Chem Inf Model 2008;48;489-97.

[37] Zhang L, Lin D, Sun X, Curth U, Drosten C, Sauerhering L, et al. Crystal structure of SARS-CoV-2 main protease provides a basis for design of improved $\alpha$-ketoamide inhibitors. Science 2020;368;409-12.

[38] Jin Z, Du X, Xu Y, Deng Y, Liu M, Zhao Y, et al. Structure of $\mathrm{M}^{\mathrm{p} r o}$ from SARS-CoV-2 and discovery of its inhibitors. Nature 2020;582;289-93.

[39] Wrapp D, Wang N, Corbett KS, Goldsmith JA, Hsieh CL, Abiona O, et al. Cryo-EM structure of the 2019-nCoV spike in the prefusion conformation. Science $2020 ; 367 ; 1260-3$. 